\title{
DERIVATIONS INTO THE INTEGRAL CLOSURE
}

BY

\author{
RICHARD DRAPER AND KLAUS FISCHER
}

\begin{abstract}
Let $A$ be a reduced analytical $k$-algebra of dimension $r$ and $A^{\prime}$ its integral closure in the full ring of quotients of $A$. We investigate the condition on $A$ that there exist $r$ elements $x_{1}, \ldots, x_{r}$ in $A$ and $k$-derivations $d_{1}, \ldots, d_{r}$ from $A$ into $A^{\prime}$ so that $d_{i}\left(x_{j}\right)$ is the $r \times r$ identity matrix and so that $d_{1}, \ldots, d_{r}$ freely generate $\operatorname{Der}_{k}\left(A, A^{\prime}\right)$. We show this is equivalent to a number of other conditions. If $A$ is a complete intersection, then the above is equivalent to the Jacobian ideal $J$ becoming principal in $A^{\prime}$. If $A / \sqrt{J}$ is regular of dimension $r-1$ and satisfies the above condition, then $A^{\prime}$ is regular.
\end{abstract}

Introduction. Let $A$ be a reduced analytic $k$-algebra. That is, $A$ is of the form $k\left[\left[x_{1}, \ldots, x_{n}\right]\right] / I$ where $I$ is a radical ideal. We shall assume that $k$ is a field of characteristic zero. Let $A^{\prime}$ be the integral closure of $A$ in its full ring of quotients. We consider two related but not equivalent conditions that we may impose on $A$ and which arise often in singularity theory. These conditions deal with $k$-derivations from $A$ into $A^{\prime}$. Of course, much is known about $k$-derivations from $A$ into $A$ and our results are motivated by and generalize this case.

If $\Omega_{A / k}$ is the module of finite differentials of $A$ over $k$, then we denote by $J$ the smallest nonzero Fitting ideal of $\Omega_{A / k}$. If $A$ is pure dimensional then $J$ is the Jacobian ideal of $A$ and the prime ideals $q$ of $A$ that do not contain $J$ are precisely those for which $A_{q}$ is a regular ring (see [B, Satz 3.2.2, p. 91]). The two conditions which we study in this paper are the following.

Condition I. $J A^{\prime}$ is a principal ideal in $A^{\prime}$ generated by a regular element.

Condition II. There exist $r$ elements $x_{1}, \ldots, x_{r}$ and $k$-derivations $d_{1}, \ldots, d_{r}$ from $A$ into $A^{\prime}$ such that $d_{i}\left(x_{j}\right)$ is the $r \times r$ identity matrix and such that $d_{1}, \ldots, d_{r}$ freely generate $\operatorname{Der}_{k}\left(A, A^{\prime}\right)$ as an $A^{\prime}$-module.

In $\$ 1$ we show that Condition I implies Condition II and that the two are equivalent if $A$ is a complete intersection. Both conditions assure that $A$ is of pure Krull dimension $r$ (Theorem 4).

Versions of these conditions appear in three forms in the literature. The first is with $A^{\prime}$ replaced by $A$. If this is so, then Condition I states that $J A$ is principal generated by a regular element and this is the version of the Jacobian criterion

Received by the editors December 1, 1980 and, in revised form, March 12, 1981 and April 6, 1981. Presented on November 5, 1981 at a Conference on Commutative Algebra at Virginia Commonwealth University.

1980 Mathematics Subject Classification. Primary 14B05, 13B10; Secondary 32B30, 13 B20.

Key words and phrases. Derivations, integral closure, reduced analytical $k$-algebra, Jacobian ideal, complete intersection, ramified primes. 
( $J A=A$ ) studied by $\mathrm{J}$. Lipman in [L2]. In that paper he proved that if $A$ is the local ring of a $k$-variety at a (not necessarily closed) point, then $J A$ principal with regular generator is equivalent to $A$ being regular ( $k$ of characteristic zero). In fact, inspection of [L2] shows that he proved that Condition I implies Condition II for arbitrary local rings ( $A^{\prime}$ still replaced by $A$ ). (See [L2, Lemmas 1 and 2] and our Conditions III and IV below.) Condition II in this case can be used to prove the regularity of $A$ (Zariski; cf. Theorem 11) and, of course, if $A$ is regular then the partial derivatives on $k\left[\left[x_{1}, \ldots, x_{r}\right]\right] \cong A$ yield the derivations of Condition II. Therefore, if $A^{\prime}$ is replaced by $A$, then both conditions are equivalent to the regularity of $A$.

The second way Conditions I and II appear is in equisingularity theory. If $A$ is a hypersurface and $A / \sqrt{J}$ is regular of dimension $r-1$ then Condition I is precisely the Jacobian criterion of equisingularity developed by Zariski [Z2]. In this case, it follows among other things that $A^{\prime}$ is a regular ring, i.e. the localizations at maximal primes are regular local rings.

The third way is in the case that $A$ is the local ring of a reduced complex analytic space $V$ at a point $p$. In another paper [DF] we prove that Condition III below (which we interpret in terms of weakly holomorphic vector fields in the complex analytic case) is equivalent to the condition that the tangent cone $C_{4}(V, p)$ defined by Whitney [W] has dimension $r$. It follows from a theorem of Stutz [S] that the normalization of $V$ is nonsingular over $p$ provided the singular set of $V$ is smooth at $p$. Stutz's proof uses the Riemann Removable Singularities Theorem.

This paper is inspired by the facts cited above. Our objectives are to examine the relationship between Conditions I and II, to determine the algebraic consequences of Condition II analogous to those found by Lipman and, given additional hypotheses on the singular locus of $A$, to obtain algebraic versions of Stutz's result.

In §1 we study the relationship between Conditions I and II. In addition to the results mentioned above we adapt arguments from [L2] to show that Condition II is equivalent to two other conditions. Let $\Omega^{\prime}$ denote the $A^{\prime}$-module $\Omega_{A / k} \otimes_{A} A^{\prime}$. (This is not the module of differentials of $A^{\prime}$ over $k$.) Let $t\left(\Omega^{\prime}\right),\left(\Omega^{\prime}\right)^{*}$, and $\left(\Omega^{\prime}\right)^{* *}$ be the torsion submodule, the dual, and the double dual, respectively (treated as $A^{\prime}$ modules). We show that Conditions II, III, and IV are equivalent.

Condition III. $\left(\Omega^{\prime}\right)^{*}$ is free of rank $r$ and the canonical mapping $\Omega^{\prime} \rightarrow\left(\Omega^{\prime}\right)^{* *}$ is surjective,

Condition IV. $\Omega^{\prime} / t\left(\Omega^{\prime}\right)$ is $A^{\prime}$-free of rank $r$.

$\S 2$ is somewhat technical and lays ground work for $\S 3$. We show that if $A$ satisfies Condition II, then by a number of coordinate changes on the ambient space, we may assume that $x_{1}, \ldots, x_{r}$ form part of a minimal basis for the maximal ideal of $A$ and also a system of parameters for $A$. Consequently, $A$ is finitely generated over $R=k\left[\left[x_{1}, \ldots, x_{r}\right]\right]$. The latter is a regular local ring since the $x_{i}$ 's are seen to be analytically independent over $k$.

In $\S 3$ we study the singular locus, the nonnormal locus, and the ramification locus of $A$ over $R$. Assuming Condition II and that coordinates (hence $R$ ) are chosen as in $\S 2$, we show that these loci agree. The fact that the ramification locus of $A$ over $R$ 
agrees with the singular locus of $A$ is very useful. It enables us to formulate strong results about $A$ and $A^{\prime}$. In particular, if $k$ is algebraically closed and $A$ satisfies Condition II then the codimension of $J$ must be less than or equal to one except in those "trivial" cases where each of the irreducible components of $A$ are already regular rings. That is, if $A$ satisfies Condition II and has a singular set of codimension greater than 1 and $k$ is algebraically closed then $A^{\prime}$ is regular. (This is somewhat stronger than the analogous result of Stutz [S, 4.2] where he assumed the singular locus to be regular even in case of high codimension.) Furthermore, a theorem by Zariski [Z1] allows us, by considering the ramification locus of $A$ over $R$, to extend regular derivations on $R$ to regular derivations on $A^{\prime}$. Using this we show that if $A$ satisfies Condition II and $A / \sqrt{J}$ is regular of dimension $r-1$ then $A^{\prime}$ is regular. (As a consequence the regularity of $A^{\prime}$ in the equisingular case follows as a corollary.) This gives us a theorem analogous to the theorem of Stutz mentioned in the beginning of this Introduction. It appears that we have used the technique of extending derivations from $R$ to $A^{\prime}$ to replace his use of the Riemann Removable Singularities Theorem although our proof is by no means a translation of his into algebraic language.

1. Relationship between Conditions I and II. We begin with some generalities that recast Lemmas 1 and 2 of [L2] in a form convenient for our use.

Let $S$ be a Noetherian ring (commutative and with an identity element) and let $M$ be a finitely generated $S$-module. If $M$ is generated by $e_{1}, \ldots, e_{n}$, let $\left(a_{i j}\right), i=$ $1, \ldots, m, j=1, \ldots, n$, be the matrix with entries in $S$ whose rows generate all the relations of $e_{1}, \ldots, e_{n}$, i.e. $\Sigma_{j} a_{i j} e_{j}=0, i=1, \ldots, m$, in $M$. For $r \geqslant 0$, the $r$ th Fitting ideal of $M$, denoted $I_{r}(M)$, is the ideal in $S$ generated by the $(n-r) \times(n-r)$ minors of $\left(a_{i j}\right)$. By convention, $I_{r}(M)=S$ for $r \geqslant n$.

We denote by $t(M)$ the torsion submodule of $M$ which consists of those elements of $M$ that are annihilated by a regular element of $S$. The smallest length of a projective resolution of $M$ will be denoted by $\operatorname{dim}_{\operatorname{proj}_{S}}(M)$.

Lemma 1. Let $S$ be a semilocal, Noetherian ring and let $M$ be a nonzero, finitely generated $S$-module. The following are equivalent.

(i) The smallest nonzero Fitting ideal of $M$ is $I_{r}(M)$ and it is principal with regular generator.

(ii) $\operatorname{dim} \operatorname{proj}_{S}(M) \leqslant 1$ and $M / t(M)$ is free of rank $r$.

Proof. The proof that (ii) implies (i) is exactly the same as the proof in [L2]. To prove (i) implies (ii) let $\mathrm{m}$ be a maximal ideal of $S$. Then $I_{r} S_{\mathrm{m}}=I_{r}\left(M_{\mathrm{m}}\right)$. It is the smallest nonzero Fitting ideal of $M_{\mathrm{m}}$, and it is principal with regular generator. (The regular generator of $I_{r}(M)$ cannot go to zero in $S_{\mathrm{m}}$.) By Lemma 1 of [L2], $M_{\mathrm{m}}$ has projective dimension less than or equal to one and $M_{\mathrm{m}} / t\left(M_{\mathrm{m}}\right)$ is free of rank $r$. Since torsion commutes with localization and a module locally free of rank $r$ over a semilocal ring is globally free of rank $r$ [Si, Theorems 5.2 and 5.4], it follows that

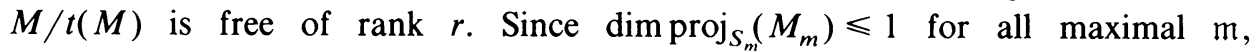
$\operatorname{dim} \operatorname{proj}_{S}(M) \leqslant 1[$ Nc, p. 188, Theorem 11]. Q.E.D. 
LEMma 2. Let $S$ be a reduced Noetherian ring and let $M$ be a finitely generated $S$-module. The following are equivalent.

(i) $M / t(M)$ is $S$-free of rank $r$.

(ii) $M^{*}=\operatorname{Hom}_{S}(M, S)$ is $S$-free of rank $r$ and the canonical homomorphism $f$ : $M \rightarrow M^{* *}$ is surjective.

Furthermore, they imply that $M \otimes_{S} Q(S)$ is $Q(S)$-free of rank $r$ where $Q(S)$ denotes the total quotient ring of $S$.

Proof. The equivalence of (i) and (ii) follows from Lemma 2 of [L2] provided we show that (ii) implies $M \otimes_{S} Q(S)$ is $Q(S)$-free of rank $r$. This follows from the fact that $S$ reduced and Noetherian implies $Q(S)$ is semisimple. Let $N=M \otimes_{S} Q(S)$, then $t(N)=\operatorname{Ker}\left(N \rightarrow N^{* *}\right)$ where duality is as $Q(S)$ module. $Q(S)$ has no regular nonunits so $t(N)=0$ and $N \simeq N^{* *}$. This is free of rank $r$ because $N^{*} \simeq$ $\operatorname{Hom}_{S}(N, S) \otimes_{S} Q(S)$ is free of rank $r$. Q.E.D.

Hereafter our data will be as described in the Introduction. $A$ will be a reduced analytic $k$-algebra of characteristic zero.

Let $p_{1}, \ldots, p_{k}$ be the minimal primes of $A . Q\left(A / p_{i}\right)=A_{p_{i}}=F_{i}$ is a field and $Q(A)=\oplus F_{i}$. In $Q(A), 1=\Sigma_{i} e_{i}$ and $F_{i}=e_{i} Q(A)$. If we denote by $A_{i}^{\prime}$ the integral closure of $A / p_{i}$ in $F_{i}$ then $A^{\prime}=A_{1}^{\prime} \oplus \cdots \oplus A_{h}^{\prime}$ and we have a commutative diagram where $A^{\prime}$ is the integral closure of $A$ in $Q(A)$ :

$$
\begin{array}{ccc} 
& A^{\prime}=A_{1}^{\prime} \oplus \cdots \oplus A_{h}^{\prime} \\
& i \nearrow & \uparrow \\
A \quad & \rightarrow & A / p_{1} \oplus \cdots \oplus A / p_{h}
\end{array}
$$

Here, $i$ is the inclusion map, $E(a)=e_{1} a \cdots e_{h} a$, and $e_{i} A=A / p_{i}$.

Lemma 3. Let $A$ be a reduced analytic k-algebra of characteristic zero. Then $A$ has pure dimension $r$ if and only if $\Omega \otimes_{A} Q(A)$ is $Q(A)$-free of rank $r$.

Proof. If $N$ is any $Q(A)$-module then $N=\bigoplus e_{i} N$ and each $e_{i} N$ is an $F_{i}$-vector space. It is straightforward to show that $N$ is $Q(A)$-free of rank $r$ if and only if each $e_{i} N$ is $F_{i}$-free of rank $r$.

Now set $N=\Omega \otimes_{A} Q(A)$. In this case

$$
e_{i} N \simeq \Omega \otimes_{A} A_{p_{i}} \simeq \Omega\left(A_{p_{i}}\right)=\Omega\left(Q\left(A / p_{i}\right)\right) \simeq \Omega\left(A / p_{i}\right) \otimes_{A / p_{i}} Q\left(A / p_{i}\right) .
$$

Therefore, $e_{i} N$ is $F_{i}$-free of rank $r$ if and only if $\Omega\left(A / p_{i}\right)$ has rank $r$ as an $A / p_{i}$-module. But $A$ reduced and $k$ of characteristic zero implies that $A$ is separable [B, Folgerung 3.1.4]. This means that the rank of $\Omega\left(A / p_{i}\right)$ is equal to $\operatorname{dim}\left(A / p_{i}\right)$ for all $i$. Q.E.D.

THEOREM 4. Let $A$ be a reduced, analytic k-algebra of characteristic zero and integral closure $A^{\prime}$. Let $\Omega$ denote the module of $k$-differentials of $A, J$ the smallest nonzero Fitting ideal of $\Omega$, and $\Omega^{\prime}=\Omega \otimes_{A} A^{\prime}$. Conditions (II), (III) and (IV) below are equivalent and are implied by (I). They imply that $A$ has pure dimension $r$. Furthermore, if $A$ is a complete intersection then they are all equivalent.

(I) $J$ is the rth Fitting ideal of $A$ and $J A^{\prime}$ is principal with regular generator. 
(II) There exist $d_{1}, \ldots, d_{r}$ in $\operatorname{Der}_{k}\left(A, A^{\prime}\right)$ and $x_{1}, \ldots, x_{r}$ in $A$ such that $d_{i} x_{j}=\delta_{i j}$ and $d_{1}, \ldots, d_{r}$ freely generate $\operatorname{Der}_{k}\left(A, A^{\prime}\right)$ over $A^{\prime}$.

(III) $\left(\Omega^{\prime}\right)^{*}$ is $A^{\prime}$-free of rank $r$ and there exist nonunits $x_{1}, \ldots, x_{r}$ in the maximal ideal of $A$ such that $\left(\Omega^{\prime}\right)^{* *}$ is $A^{\prime}$-generated by the images of $d x_{1} \otimes 1, \ldots, d x_{r} \otimes 1$ under the canonical map $\Psi: \Omega^{\prime} \rightarrow\left(\Omega^{\prime}\right)^{* *}$.

(IV) $\Omega^{\prime} / t\left(\Omega^{\prime}\right)$ is $A^{\prime}$-free of rank $r$.

Proof. Lemma 1 gives that (I) implies (IV). Lemmas 2 and 3 show that (IV) implies $A$ has pure dimension $r$ since $Q\left(A^{\prime}\right)=Q(A), A^{\prime}$ is reduced, and $\Omega^{\prime} \otimes_{A^{\prime}} Q\left(A^{\prime}\right)$ $=\Omega \otimes_{A} Q(A)$. The equivalence of (IV) and (III) follows from Lemma 2 if we show that the condition $\left(\Omega^{\prime}\right)^{*}$ is $A^{\prime}$-free of rank $r$ and the map $\Psi$ onto implies the existence of the $x_{i}$ as specified in (III).

Let $y_{1}, \ldots, y_{n}$ be a generating set for the maximal ideal of $A$. Then $d y_{1}, \ldots, d y_{n}$ generate $\Omega$ where $d: A \rightarrow \Omega$ is the canonical derivation. Hence, $d y_{1} \otimes 1, \ldots, d y_{n} \otimes 1$ generate $\Omega^{\prime}$. We will let $x_{i}=\sum_{j=1}^{n} a_{i j} y_{j}$ and show that $\Psi\left(d\left(x_{i}\right) \otimes 1\right), 1 \leqslant i \leqslant r$, generates $\left(\Omega^{\prime}\right)^{* *}$ for a suitable choice of the matrix $\left[a_{i j}\right]$. To further simplify notation let $w_{j}=\Psi\left(d y_{j} \otimes 1\right)$ and $z_{i}=\sum a_{i j} w_{j}$. Since $\Psi$ is onto, $w_{1}, \ldots, w_{n}$ generates $\left(\Omega^{\prime}\right)^{* *}$. Let $M$ denote $\left(\Omega^{\prime}\right)^{* *}$.

Let $\mathrm{m}$ be a maximal ideal of $A^{\prime}$ and pass to $M_{\mathrm{m}}$. By Nakayama's Lemma a set $w_{j_{1}}, \ldots, w_{j_{r}}$ is a free basis for $M$, but the set $j_{1}, \ldots, j_{r}$ depends on $m$. We establish conditions on the matrix $\left[a_{i j}\right]$ which insure that $z_{1}, \ldots, z_{r}$ is a basis for $M_{\mathfrak{m}}$ for all $\mathrm{m}$.

Let $e_{1}, \ldots, e_{r}$ be a basis for $(M)^{*} \cong\left(\Omega^{\prime}\right)^{*}$ and consider $e_{k} z_{i}=\sum a_{i j} e_{k} w_{j}$. The $n \times r$ matrix $\left[e_{k} w_{j}\right.$ ] has its elements in $A^{\prime}$ and $w_{j_{1}}, \ldots, w_{j_{r}}$ is a free basis for $M_{\mathrm{m}}$ if and only if $\left[e_{k} w_{j_{i}}\right]$ is invertible in $A_{\mathrm{m}}^{\prime}$. Therefore the matrix $\left[e_{k} w_{j}\right]$ locally has rank $r$ on $A^{\prime}$. We want to choose $\left[a_{i j}\right]$ so that $\left[e_{k} z_{i}\right]$ locally has rank $r$ on $A^{\prime}$. Let $D=\Sigma \alpha_{\lambda} \delta_{\lambda}$ denote the determinant of $\left[e_{k} z_{i}\right]$ where $\alpha_{\lambda}$ and $\delta_{\lambda}$ are determinants of $r \times r$ minors of $\left[a_{i j}\right]$ and $\left[e_{k} w_{j}\right]$, respectively. Regard $D$ as a polynomial in the entries of $\left[a_{i j}\right]$ (as they appear in the $\alpha_{\lambda}$ ) with coefficients in $A^{\prime}$. Let $K^{\prime}$ denote the residue class field of $A_{\mathrm{m}}^{\prime}$ and $\bar{D}=\Sigma \alpha_{\lambda} \bar{\delta}_{\lambda}$ the corresponding polynomial with coefficients in $K^{\prime}$. The $\bar{\delta}_{\lambda}$ corresponding to $\operatorname{det}\left[e_{k} w_{j_{i}}\right]$ is nonzero so $\bar{D}$ is not identically zero. $K^{\prime}$ is a finite algebraic extension of $k$. Let $L$ be a least normal extension of $k$ containing $K^{\prime}$ and let $G$ be the Galois group of $L$ over $k$. Let $D_{\mathrm{m}}=\prod_{g \in G}\left(\sum \alpha_{\lambda} g\left(\bar{\delta}_{\lambda}\right)\right)$. This is a polynomial in the entries of $\left[a_{i j}\right]$ which is invariant under the action of $G$ so has coefficients in $k$. It is not identically zero because $\Sigma_{\lambda} \alpha_{\lambda} g\left(\bar{\delta}_{\lambda}\right)$ is not for any $g$. Since $k$ is an infinite field there exists $\left[a_{i j}\right]$ with entries in $k$ for which $D_{\mathfrak{m}} \neq 0$ for any maximal $\mathrm{m}$ in $A^{\prime}$. For such a choice of $\left[a_{i j}\right]$ the matrix $\left[e_{k} z_{i}\right]$ locally has rank $r$ on $A^{\prime}$. It follows that $\left(z_{1}, \ldots, z_{r}\right)$ locally generates $M$, therefore $\left(z_{1}, \ldots, z_{r}\right)$ generates $M$.

Next we prove the equivalence of (III) and (II). Asterisks will denote $A^{\prime}$-duals.

$$
\begin{aligned}
\left(\Omega^{\prime}\right)^{*} & =\operatorname{Hom}_{A^{\prime}}\left(\Omega^{\prime}, A^{\prime}\right)=\operatorname{Hom}_{A^{\prime}}\left(\Omega \otimes_{A} A^{\prime}, A^{\prime}\right) \\
& \simeq \operatorname{Hom}_{A}\left(\Omega, \operatorname{Hom}_{A^{\prime}}\left(A^{\prime}, A^{\prime}\right)\right) \\
& \simeq \operatorname{Hom}_{A}\left(\Omega, A^{\prime}\right)=\operatorname{Der}_{k}\left(A, A^{\prime}\right) .
\end{aligned}
$$

Therefore (III) implies that $\operatorname{Der}_{k}\left(A, A^{\prime}\right)$ is $A^{\prime}$-free of rank $r$. Let

$$
\varphi: \operatorname{Der}_{k}\left(A, A^{\prime}\right) \rightarrow\left(\Omega^{\prime}\right)^{*}
$$


denote the isomorphism just established. If $\delta \in \operatorname{Der}_{k}\left(A, A^{\prime}\right), x \in A, \alpha \in A^{\prime}$, and $d$ : $A \rightarrow \Omega$ is the canonical derivation then $\varphi(\delta)(d x \otimes \alpha)=\alpha(\delta(x))$. Thus if $\left\{d_{i}, 1 \leqslant i\right.$ $\leqslant r\} \subset \operatorname{Der}_{k}\left(A, A^{\prime}\right)$ is chosen so that $\left\{\varphi\left(d_{i}\right), 1 \leqslant i \leqslant r\right\}$ is dual to $\left\{\Psi\left(d x_{i} \otimes 1\right)\right.$, $1 \leqslant i \leqslant r\}$, then

$$
\delta_{i j}=\varphi\left(d_{i}\right)\left(\Psi\left(d x_{j} \otimes 1\right)\right)=1 \cdot d_{i} x_{j} .
$$

This shows that (III) implies (II). Of course $d_{i} x_{j}=\delta_{i j}$ guarantees the $A^{\prime}$-independence of $\left\{d_{i}, 1 \leqslant i \leqslant r\right\}$.

If we assume (II) then the isomorphism $\varphi$ insures that $\left(\Omega^{\prime}\right)^{*}$ is $A^{\prime}$-free of rank $r$ and, therefore, $\left(\Omega^{\prime}\right)^{* *}$ is $A^{\prime}$-free of rank $r$. The condition $d_{i} x_{j}=\delta_{i j}$ together with the description of $\varphi$. show that $\left\{\Psi\left(d x_{j} \otimes 1\right), 1 \leqslant j \leqslant r\right\}$ is a dual basis to the basis $\left\{\varphi\left(d_{i}\right), 1 \leqslant i \leqslant r\right\}$ for $\left(\Omega^{\prime}\right)^{*}$. (If $\omega_{1}, \ldots, \omega_{r}$ is a basis for $\left(\Omega^{\prime}\right)^{* *}$ then $d x_{j} \otimes 1 \rightarrow \Sigma_{i} a_{i j} \omega_{i}$, $a_{i j} \in A^{\prime}$, and $\varphi\left(d_{k}\right)\left(d x_{j} \otimes 1\right)=\delta_{k j}=\sum_{i} a_{i j} \omega_{i}\left(\varphi\left(d_{k}\right)\right)$. Since $\left\{\varphi\left(d_{k}\right), 1 \leqslant k \leqslant r\right\}$ is a basis for $\left(\Omega^{\prime}\right)^{*}$ and $\left\{\omega_{i}, 1 \leqslant i \leqslant r\right\}$ is a basis for $\left(\Omega^{\prime}\right)^{* *}$, the matrix $\omega_{i}\left(\varphi\left(d_{k}\right)\right)$ is invertible in $A^{\prime}$. Therefore the matrix $\left[a_{i j}\right]$ is invertible in $A^{\prime}$ so $\left\{\Psi\left(d x_{j} \otimes 1\right)\right.$, $1 \leqslant j \leqslant r\}$ is a basis for $\left(\Omega^{\prime}\right)^{* *}$.)

Finally we prove (IV) implies (I) if $A$ is a complete intersection in which case $\operatorname{dim} \operatorname{proj}_{A}(\Omega) \leqslant 1$ [B, Satz 3.5.3]. Therefore, if (IV) holds, (I) will follow from Lemma 1 if we show $\operatorname{dim}_{\operatorname{proj}_{A^{\prime}}}\left(\Omega^{\prime}\right) \leqslant 1$. We have $0 \rightarrow F^{n-r} \rightarrow F^{n} \rightarrow \Omega \rightarrow 0$ with the $F^{i}$ free of the indicated rank by Lemma 3. By [L1, Lemma, p. 889] $F^{n-r} \rightarrow F^{n}$ is injective if and only if $[0: J]=0$, i.e. $J$ contains a regular element. A regular element on $A$ remains regular on $A^{\prime}$ because $A$ is reduced. Using the same reference we conclude that $0 \rightarrow F^{n-r} \otimes A^{\prime} \rightarrow F^{n} \otimes A^{\prime} \rightarrow \Omega^{\prime} \rightarrow 0$ is exact. Q.E.D.

We are indebted to the referee who suggested significant simplifications of our original proof of Theorem 4 .

The theorem shows the implications concerning the conditions as stated in the Introduction. The following example shows that in general, Condition II does not imply Condition I.

EXAMPLE. Let $V$ be the image of $k^{2}$ under the mapping $(r, s) \rightarrow\left(r, s^{3}, s^{4}, r s^{5}\right)=$ $(x, y, u, v)$. Consider the germ of $V$ at the origin. Let $A=k[[x, y, u, v]] / P$ be its structure ring. $A$ has the following properties.

(i) $A^{\prime}=k[[r, s]]$.

(ii) $P$ is generated by $v^{2}-x^{2} y^{2} u, v y-x u^{2}, u v-x y^{3}$, and $u^{3}-y^{4}$.

(iii) $J A^{\prime}$ is generated by $r s^{6}$ and $s^{8}$.

(iv) $\operatorname{Der}_{k}\left(A, A^{\prime}\right)$ is free of rank 2 with basis $d_{1}=\left(1,0,0, s^{5}\right)$ and $d_{2}=$ $\left(0,3 / 5,4 s / 5, r s^{2}\right)$.

(v) $\Omega \otimes_{A} A^{\prime}$ is not free.

It follows from (iii) that $J A^{\prime}$ is not principal and from (iv) that $\left\{d_{1}, d_{2}\right\},\{x, 5 y / 3\}$ satisfy (II) of Theorem 4. That is, this example satisfies Condition II but not Condition I. This ring has some other interesting properties.

(vi) $A$ is not Cohen-Macaulay.

(vii) $J$ has an embedded associated prime.

(viii) $J A^{\prime}$ has an embedded associated prime.

(ix) The conductor is unmixed on $A^{\prime}$. 
The proofs are easy. Modulo $r$, every element of $A$ becomes a zero divisor so depth $A=1 . J=\left(s^{12}, s^{11}, r s^{13}, r^{2} s^{10}, r s^{8}, r^{2} s^{9}\right)$. Its associated primes are $\left(s^{3}, s^{4}, r s^{5}\right)$ and the maximal ideal. To see this observe that $s^{8}$ modulo $J$ is annihilated by the maximal ideal. $J A^{\prime}=\left(r s^{8}, s^{11}\right)$ has $(s)$ and the maximal ideal of $A^{\prime}$ for associated primes. To see the latter is associated observe that $s^{10}$ is annihilated by the maximal ideal modulo $J A^{\prime}$. The conductor is $\left(s^{6}\right)$.

2. Further remarks on Condition II. If $A$ is a reduced Noetherian ring with integral closure $A^{\prime}$ in its full ring of quotients $Q(A)$, then any derivation of $A$ into $A^{\prime}$ may be viewed as the sum of derivations along the components of $A$. Specifically, we have as in $\$ 1$ the commutative diagram:

$$
\begin{aligned}
& A^{\prime}=A_{1}^{\prime} \oplus \cdots \oplus A_{h}^{\prime} \\
& \begin{array}{ccc} 
& i \nearrow & \uparrow \\
A & \stackrel{E}{\rightarrow} & A / p_{1} \oplus \cdots \oplus A / p_{k}
\end{array}
\end{aligned}
$$

Recall that $i$ is an injection and $E(a)=e_{1} a \oplus \cdots \oplus e_{h} a$ where the $e_{i}$ 's are the orthogonal elements in the decomposition of $A^{\prime}$.

Let $k$ be a subfield of $A$.

Lemma 5. Suppose $d$ is a $k$-derivation of $A$ to $A^{\prime}$. Then $d$ induces a $k$-derivation $\tilde{d}_{i}$ of $A / p_{i}$ to $\left(A / p_{i}\right)^{\prime}=A_{i}^{\prime}$ so that $e_{i} d=\tilde{d}_{i} E$. Hence, $d$ induces a $k$-derivation $\tilde{d}=\tilde{d}_{1}$ $\oplus \cdots \oplus \tilde{d}_{h}$ so that $d=\tilde{d} E$. Conversely, given a collection of $k$-derivations $\tilde{d}_{i}, i=$ $1, \ldots, h$, of $A / p_{i}$ to $A_{i}^{\prime}, \tilde{d}=\tilde{d}_{i} \oplus \cdots \oplus \tilde{d}_{h}$ is a $k$-derivation of $A / p_{i} \oplus \cdots \oplus A / p_{h}$ to $A^{\prime}$ which gives rise, via restriction to $A$, of a $k$-derivation $d$ from $A$ to $A^{\prime}$.

Proof. Let $\mathscr{P}_{i}$ be the (unique) prime in $A^{\prime}$ lying over $p_{i}$, i.e. $p_{i}=A \cap \mathscr{P}_{i}$. Then $A / p_{i}$ is identified in $A^{\prime} / \mathscr{P}_{i}=\left(A / p_{i}\right)^{\prime}$. Since $0=p_{1} \cap \cdots \cap p_{h}$ is an irredundant representation we may choose $z_{i} \in \bigcap_{j \neq i} p_{j}$ and $z_{i} \notin p_{i}$. It follows that $p_{i}=\left(0: z_{i}\right)$. If $x \in p_{i}$, then $x z_{i}=0$ and hence

$$
d\left(x z_{i}\right)=x d\left(z_{i}\right)+z_{i} d(x)=0 .
$$

But $x d\left(z_{i}\right) \in \mathscr{P}_{i}$. Since $z_{i} \notin p_{i}$, it does not belong to $\mathscr{P}_{i}$ and hence $d(x) \in \mathscr{P}_{i}$. Hence $d\left(p_{i}\right) \subset \mathscr{P}_{i}$ and we see that it induces a derivation of $A / p_{i}$ to $A_{i}^{\prime}$. The rest of the first part of the lemma now follows directly.

Conversely, it is easy to check that a collection of $k$-derivations $\tilde{d}_{i}, i=1, \ldots, h$, gives. a $k$-derivation of $A / p_{1} \oplus \cdots \oplus A / p_{h}$ and the rest follows. Q.E.D.

We note that the lemma in fact shows that $e_{i} \operatorname{Der}_{k}\left(A, A^{\prime}\right) \cong \operatorname{Der}_{k}\left(A / p_{i},\left(A / p_{i}\right)^{\prime}\right)$ and hence $\operatorname{Der}_{k}\left(A, A^{\prime}\right) \cong \oplus \operatorname{Der}_{k}\left(A / p_{i},\left(A / p_{i}\right)^{\prime}\right)$ as $A^{\prime}$-modules.

LemMa 6. If $A$ is reduced and if $A$ satisfies Condition II, then so do all the irreducible components of $A$.

Proof. Let $x_{1}, \ldots, x_{r}$ and $d_{1}, \ldots, d_{r}$ be the elements and derivations arising from the assumption that $A$ satisfies Condition II. Hence, $d_{i}\left(x_{j}\right)$ is the unit matrix in $A^{\prime}$ and $d_{1}, \ldots, d_{r}$ generate $\operatorname{Der}_{k}\left(A, A^{\prime}\right)$. But the preceding lemma and the subsequent decomposition of $\operatorname{Der}_{k}\left(A, A^{\prime}\right)$ assure that for each $j=1, \ldots, h$, the elements $e_{j} x_{i}$ and the derivations $e_{j} d_{i}$ also satisfy Condition II on $A / p_{j}$. Q.E.D. 
The above now gives another way of characterizing Condition II (cf. [L1, Theorem 3]).

LemMa 7. Let $A$ be a reduced analytic $k$-algebra of characteristic zero with integral closure $A^{\prime}$ and suppose that $A$ is pure dimensional. Suppose that there exists a system of parameters $\left(x_{1}, \ldots, x_{r}\right)$ and $d_{i} \in \operatorname{Der}_{k}\left(A, A^{\prime}\right), 1 \leqslant i \leqslant r$, such that $d_{i}\left(x_{j}\right)=\delta_{i j}$. Then if $d \in \operatorname{Der}_{k}\left(A, A^{\prime}\right), d=d\left(x_{i}\right) d_{i}$ and $d_{1}, \ldots, d_{r}$ freely generate $\operatorname{Der}_{k}\left(A, A^{\prime}\right)$ as $A^{\prime}$ module.

Proof. Let $p$ be any of the associated primes of 0 . Since $A$ is pure dimensional, any system of parameters for $A$ must have their image in $A / p$ be a system of parameters for $A / p$. It follows from the decomposition of Lemma 5 that we may assume at the outset that $A$ is a domain.

The elements $x_{1}, \ldots, x_{r}$ are analytically independent over $k$ so $A$ contains $R=$ $k\left[\left[x_{1}, \ldots, x_{r}\right]\right]$. Furthermore, every element of $A$ is integral over $R$. See [N, 31.6]. The condition $d_{i} x_{j}=\delta_{i j}$ insures that $d_{1}, \ldots, d_{r}$ are $A^{\prime}$-independent. The object is to show that they $A^{\prime}$-generate $\operatorname{Der}_{k}\left(A, A^{\prime}\right)$. We claim that for $d \in \operatorname{Der}_{k}\left(A, A^{\prime}\right)$,

$$
d=\sum_{i=1}^{r} d\left(x_{i}\right) d_{i} .
$$

Let $e$ denote the right-hand derivation. To prove the claim take $y \in A$. It satisfies an integral equation

$$
f(y)=y^{s}+r_{1} y^{s-1}+\cdots+r_{s}=0
$$

with $r_{i} \in R$. Then

$$
d(f(y))=f^{\prime}(y) d(y)+f^{d}(y)=0
$$

where $f^{d}$ is the result of applying $d$ to the coefficients of $f$. But $f^{d}(y)$ is readily seen to equal $f^{e}(y)$ and applying $e$ yields

$$
e(f(y))=f^{\prime}(y) e(y)+f^{e}(y)=0 .
$$

Choosing $f$ to be the minimal polynomial for $y$ over $R$ insures $f^{\prime}(y) \neq 0$. The above equations show $e(y)=d(y)$. Q.E.D.

The next few results, needed for the final section, record specific conclusions that may be drawn about the elements $x_{1}, \ldots, x_{r}$ arising in Condition II. Similar results may be obtained if the ring satisfies Condition I.

LEMMA 8. Let $(A, M)$ be a reduced analytic $k$-algebra of characteristic zero with integral closure $A^{\prime}$. Suppose that $x_{i}$ are nonunits and $d_{j}-\operatorname{Der}_{k}\left(A, A^{\prime}\right)$ with $d_{j}\left(x_{i}\right)=\delta_{i j}$, $1 \leqslant i, j \leqslant r$. Suppose $\left(z_{1}, \ldots, z_{n}\right)$ is a minimal set of generators for $M$. Then for $a$ generic choice of $\left[c_{j k}\right] \subset k^{n \times n}$, we may find a minimal set of generators $y_{1}, \ldots, y_{n}$ where $y_{j}=\Sigma c_{j k} z_{k}, 1 \leqslant j \leqslant n$, and $k$-derivations $e_{i} \in \operatorname{Der}_{k}\left(A, A^{\prime}\right)$ so that $e_{i} y_{j}=\delta_{i j}, 1 \leqslant i$, $j \leqslant r$.

Proof. First we establish that there is a minimal set of generators $y_{1}, \ldots, y_{n}$ for $M$ and derivations $e_{1}, \ldots, e_{r}$ with $e_{i} y_{j}=\delta_{i j}, 1 \leqslant i, j \leqslant r$. Then we establish that this property is preserved by a generic family of matrices in $k^{n \times n}$. Let $z_{1}, \ldots, z_{n}$ be a minimal set of generators for $M$. 


$$
x_{i}=\sum_{j=1}^{n} a_{i j} z_{j}, \quad 1 \leqslant i \leqslant r, a_{i j} \in A .
$$

Since $d_{i}$ are $k$-derivations we may assume all $x_{i} \in M$. Let $a_{i j}=a_{i j}^{0}+b_{i j}$ where $a_{i j}^{0} \in k$ and $b_{i j} \in M$.

$$
\delta_{k i}=d_{k}\left(x_{i}\right)=\sum_{j=1}^{n} a_{i j}^{0} d_{k}\left(z_{j}\right)+d_{k}\left(\sum_{j=1}^{n} b_{i j} z_{j}\right) .
$$

Since $b_{i j} z_{j} \in M^{2}, d_{k}\left(M^{2}\right) \subset M A^{\prime} \subset \cap M_{\lambda} A^{\prime}$, the Jacobson radical of $A^{\prime}$. Therefore

$$
\delta_{k i}=\sum_{j=1}^{n} a_{i j}^{0} d_{k}\left(z_{j}\right) \bmod M_{\lambda}, \quad 1 \leqslant i, k \leqslant r .
$$

It follows that $\left[a_{i j}^{0}\right] \in k^{r \times n}$ is of rank $r$ and $\left[d_{k}\left(z_{j}\right)\right] \in\left(A^{\prime}\right)^{n \times r}$ is of rank $r$. Let $y_{i}=\sum_{j=1}^{n} a_{i j}^{0} z_{j}, 1 \leqslant i \leqslant r$.

$$
d_{k}\left(y_{i}\right)=\sum_{j=1}^{n} a_{i j}^{0} d_{k}\left(z_{j}\right), \quad 1 \leqslant i, k \leqslant r
$$

is invertible in $A^{\prime}$. Let $\left[b_{m k}\right]$ be its inverse and define $e_{m}=\sum_{k=1}^{r} b_{m k} d_{k}, 1 \leqslant m \leqslant r$.

$$
e_{m}\left(y_{i}\right)=\delta_{m i} \text {. }
$$

Complete $\left[a_{i j}^{0}\right]$ to $\left[c_{i j}\right]$, a nonsingular matrix in $k^{n \times n}$. Let $y_{i}=\sum_{j=1}^{n} c_{i j} z_{j}, 1 \leqslant i \leqslant n$, and we have the existence of a minimal generating set for $M$ and derivations as required by the lemma. Moreover, it is clear from the above argument that if $\left(z_{1}, \ldots, z_{n}\right)$ is a minimal generating set for $M$ and $d_{i} z_{j}=\delta_{i j}, 1 \leqslant i, j \leqslant r$, to start with then $y_{i}=\sum_{j=1}^{n} c_{i j} z_{j}$ and $e_{i}$ satisfy the conclusion of the lemma if rank $\left[c_{i j}\right]=n$, $\operatorname{rank}\left[c_{i j}\right]=r, 1 \leqslant i, j \leqslant r$, and $e_{i}=\sum_{i=1}^{r} b_{i j} d_{j}$ with $\left[b_{i j}\right]=\left[c_{i j}, 1 \leqslant i, j \leqslant r\right]^{-1}$. This shows that the condition is generic. Q.E.D.

We now introduce the condition $A / \sqrt{J}$ regular of dimension $r-1$.

Lemma 9. Suppose $(A, M)$ is a reduced analytic $k$-algebra of pure dimension $r$ and characteristic zero. Let $J$ be its Jacobian ideal and assume that $A / \sqrt{J}$ is regular of dimension $r-1$. If $\left(y_{1}, \ldots, y_{n}\right)$ is a minimal basis for $M$ and $x_{i}=\sum_{j=1}^{n} a_{i j} y_{j}$, $1 \leqslant i \leqslant n, a_{i j} \in k$, then for generic choice of $\left[a_{i j}\right] \in k^{n \times n}$, (i) $\left(x_{1}, \ldots, x_{n}\right)$ is a minimal basis for $M$ and (ii) $\left(x_{1}, \ldots, x_{r-1}\right)$ map onto a minimal basis for the maximal ideal of $A / \sqrt{J}$.

Proof. Let $[a]$ denote the $n \times n$ matrix $a_{i j}$. Insisting that $[a]$ be nonsingular insures (i) because $n$ was the dimension of $M / M^{2}$. We ask what conditions [a] must satisfy to insure (ii).

Let $B=A / \sqrt{J}$. The surjection $A \rightarrow B$ induces a $k$-surjection $M / M^{2} \rightarrow N / N^{2}$. We use the same symbol to denote an element of $A$, its image in $B, M / M^{2}$, and $N / N^{2}$. Since $\left(y_{1}, \ldots, y_{n}\right)$ is a basis for $M / M^{2}$ we can choose a basis for $N / N^{2}$ of the form $y_{i}, \rho=1, \ldots, r-1$. In $N / N^{2}$ we get relations $y_{i}=\Sigma_{\rho} c_{\rho i} y_{i_{\rho}}, 1 \leqslant i \leqslant n, c_{\rho i} \in k$. Carrying the coordinate change $x_{j}=\Sigma a_{i j} y_{i}$ of $M / M^{2}$ over to $N / N^{2}$ we have

$$
x_{j}=\sum_{i} a_{i j} \sum_{\rho} c_{\rho i} y_{i_{\rho}}=\sum_{\rho}\left(\sum_{i} c_{\rho i} a_{i j}\right) y_{i_{\rho}} .
$$


To insure that $x_{1}, \ldots, x_{r-1}$ is a basis for $N / N^{2}$ we only need to insure that the first $(r-1) \times(r-1)$ minor of the $(r-1) \times n$ matrix $[c][a]$ is invertible in $k$. The determinant of that minor has the form $\Sigma \pm \gamma \alpha$ where $\gamma$ and $\alpha$ run over determinants of $(r-1) \times(r-1)$ minors $[c]$ and the first $[r-1]$ columns of $[a]$ with column indices of $[c]$ matching row indices of $[a]$. $[c]$ is determined by the choice of bases for $M / M^{2}$ and $N / N^{2}$.

$\Sigma \pm \gamma \alpha$ is a polynomial in the entries of $[a]$. We need to insure that this polynomial does not vanish identically. In columns $i_{\rho}, \rho=1, \ldots, r-1$, the matrix [c] has $I_{r-1}$. If we choose $[a]$ to have the $i_{\rho}$ th column of $I_{n}$ in column $\rho$, $\rho=1, \ldots, r-1$, and the remaining columns of $I_{n}$ placed in the remaining columns of $[a]$ then all $\alpha$ 's occurring in $\Sigma \pm \gamma \alpha$ vanish except the one with index $i_{1}, \ldots, i_{r-1}$. It has value 1 , the corresponding $\gamma$ is also 1 so there is a nonsingular choice of $[a]$ for which $\Sigma \pm \gamma \alpha$ is not zero. Therefore, it is not identically zero as a polynomial in the entries of $[a]$. Thus coordinate changes which satisfy (ii) are generic. Q.E.D.

A minimal system of generators for the maximal ideal of $A$ is called a system of local coordinates.

THEOREM 10. Suppose $A$ is a reduced analytic $k$-algebra of characteristic zero and integral closure $A^{\prime}$. Let $r$ be the dimension of $A$. If $A$ satisfies Condition II, then by a change of coordinates we may obtain elements $x_{1}, \ldots, x_{r}$ and k-derivations $d_{i}: A \rightarrow A^{\prime}$, $i=1, \ldots, r$, so that

(i) $x_{1}, \ldots, x_{r}$ is a system of parameters for $A$ and part of a system of local coordinates,

(ii) $d_{i}\left(x_{j}\right)=\delta_{i j}$, and

(iii) $d_{1}, \ldots, d_{r}$ generate $\operatorname{Der}_{k}\left(A, A^{\prime}\right)$.

If, furthermore, $A / \sqrt{J}$ is a regular ring of dimension $r-1$, then we may find a change of coordinates so that along with the above,

(iv) $x_{1}, \ldots, x_{r-1}$ form a regular system of parameters for $A / \sqrt{J}$.

Proof. By Theorem 4, $A$ is of pure dimension $r$ and by Lemma 8 we may assume the existence of elements $y_{1}, \ldots, y_{r}$ and $k$-derivations $D_{1}, \ldots, D_{r}$ of $A$ to $A^{\prime}$ so that $D_{i}\left(y_{j}\right)=\delta_{i j}$ where $y_{1}, \ldots, y_{r}$ form part of a system of local coordinates for $A$. If $n$ is the embedding dimension of $A$ we may then extend the above elements to $y_{1}, \ldots, y_{r}$, $y_{r+1}, \ldots, y_{n}$ where these now are a system of local coordinates for $A$.

If $\left(c_{i j}\right) \in k^{n \times n}$, then $x_{i}=\sum_{j=1}^{n} c_{i j} y_{j}$ for $i=1, \ldots, n$ is also a system of local coordinates as long as $\operatorname{det}\left(c_{i j}\right) \neq 0$. Applying the derivations $D_{1}, \ldots, D_{r}$ we have

$$
\operatorname{det}\left(D_{j}\left(x_{i}\right)\right)=\operatorname{det}\left(c_{i j}+\sum_{k=r+1}^{n} c_{i, k} D_{j}\left(y_{k}\right)\right), \quad i, j=1, \ldots, r .
$$

Let $M_{\lambda}, \lambda=1, \ldots, t$, be the maximal ideals of $A^{\prime}$. Then for each $\lambda, A^{\prime} / M_{\lambda}$ is a field algebraic over $k$. Denote this field by $K_{\lambda}$. The right-hand determinant is, modulo $M_{\lambda}$, a polynomial $P_{\lambda}\left(c_{i j}\right)$ with coefficients in $A^{\prime} / M_{\lambda}$. It will be assured that $\operatorname{det}\left(D_{i}\left(x_{j}\right)\right)$ is a unit in $A^{\prime}$ as long as we can find a particular choice $\left(c_{i j}\right) \in k^{n \times n}$ so that $P_{\lambda}\left(c_{i j}\right) \neq 0$ for $\lambda=1, \ldots, t$. Since for the choice of entries $\delta_{i j}, P_{\lambda}\left(\delta_{i j}\right)=1$, we see that $P_{\lambda}$ is a nonzero polynomial with coefficients in $K_{\lambda}$. It follows by the same 
argument as in Theorem 4 that we may find a nonzero polynomial $Q_{\lambda}\left(c_{i j}\right)$ with coefficients in $k$ so that $Q_{\lambda}\left(\xi_{i j}\right) \neq 0$ implies $P_{\lambda}\left(\xi_{i j}\right) \neq 0 \forall \lambda$. Since $k$ is infinite, it follows by [A1, 23.5, p. 192], that we may find a generic change of coordinates so that $x_{1}, \ldots, x_{r}$ is a system of parameters for $A$.

Part (i) of the theorem now follows immediately and so does (ii) if we let $d_{i}=\sum_{k=1}^{r} b_{i k} D_{k}, i=1, \ldots, r$, where $\left(b_{i k}\right)$ is the $(r \times r)$ left inverse of $\left(D_{i}\left(x_{j}\right)\right)$. Part (iii) follows from Lemma 7.

If now $A / \sqrt{J}$ is regular, then Lemma 9 assures that the above change of coordinates can be made so that $x_{1}, \ldots, x_{r-1}$ have images which form a regular system of parameters for $A / \sqrt{J}$. Q.E.D.

3. Ramification and the singular locus. We assume throughout this section that $A$ is a reduced analytic $k$-algebra of characteristic zero. If $A^{\prime}$ is the integral closure of $A$ in its full ring of quotients we denote by $C$ the conductor of $A^{\prime}$ into $A$. If $q$ is any prime ideal in $A$, then $A_{q}$ is integrally closed in its full ring of quotients if and only if $q$ does not contain $C$ [A2, p. 506]. It is known [L1, Proposition 2.1] that the length of a maximal $A$ sequence in $C$ is not greater than one; i.e. the depth $C \leqslant 1$. Since $A$ is reduced, $C$ must contain at least a regular element and hence depth $C=1$. (If not, then $C \subset p$ where $p$ is some associated prime of $A$. This would mean that the field $A_{p}$ is not integrally closed in its own field of quotients.)

We recall that if $A$ is pure dimensional then a prime $q$ in $A$ is regular if and only if $q$ does not contain $J$. It follows that if $q \supset C$ then $q \supset J$, since $A_{q}$ regular implies it is integrally closed in its full ring of quotients.

Assume that $A$ satisfies Condition II as stated in the Introduction. Then $A$ is of pure dimension $r$ by Theorem 4 . Denote by $d_{1}, \ldots, d_{r}$ the derivations which freely generate $\operatorname{Der}_{k}\left(A, A^{\prime}\right)$ and by $x_{1}, \ldots, x_{r}$ the elements in $A$ so that $d_{i}\left(x_{j}\right)=\delta_{i j}$. We may assume by Theorem 10 that the $x_{i}$ 's have been so chosen that $x_{1}, \ldots, x_{r}$ is a system of parameters for $A$ and that they form part of a minimal generating set for the maximal ideal of $A$. If such a choice has been made, then $x_{1}, \ldots, x_{r}$ is analytically independent over $k$ and $R=k\left[\left[x_{1}, \ldots, x_{r}\right]\right]$ is a power series ring over which $A$ is finitely generated [N]. It follows that if $Q(A)$ is the full ring of quotients of $A$, then the integral closure of $R$ in $Q(A)$, denoted by $R^{\prime}$, agrees with $A^{\prime}$. The restriction of $d_{i}$ to $R$ gives $d_{i} \mid R=\partial / \partial x_{i}$ on $R$. We continue to denote this restriction by $d_{i}$.

Suppose $B$ is a ring containing $R$ which is integral over $R$. If $q$ is a prime ideal in $B$ which lies over a prime $p$ in $R$, i.e. $p=R \cap q$, then $q$ (or $B_{q}$ ) is said to be unramified over $p$ (or $R_{p}$ ) if the following two conditions are satisfied:

(i) $B_{q} / q B_{q}$ is separable algebraic over $R_{p} / p R_{p}$.

(ii) $p B_{q}=q B_{q}$.

Otherwise, $q$ (or $B_{q}$ ) is said to be ramified over $p$ (or $R_{p}$ ).

One aim of this section is to show that under the assumption that $A$ satisfies Condition II and is reduced, the singular locus, the ramification locus over $R$, and the primes $q$ for which $A_{q}$ is not integrally closed, all agree.

We quote a theorem proved by Nagata in the domain case and generalized by Zariski which we shall use often in the sequel. For our purposes it is presented most conveniently by Lipman [L1, Theorem 2, p. 881]. 
THEOREM 11. Suppose $\theta$ is a complete semilocal ring with residue field of characteristic zero. Let $k$ be a subring of $\theta$ and suppose there exist $k$-derivations $d_{1}, \ldots, d_{s}$ of $\theta$ into $\theta$ and $x_{1}, \ldots, x_{s}$ in the Jacobson radical of $\theta$ so that the matrix $\left(d_{i}\left(x_{j}\right)\right)$ is invertible. Let $\bar{\theta}=\theta / \theta x_{1}+\cdots+\theta x_{s}$. Under these assumptions we may conclude that

(i) $\overline{\mathcal{O}}$ may be identified as a subring of $\mathcal{O}$ containing $k$,

(ii) $x_{1}, \ldots, x_{s}$ are analytically independent over $\overline{\mathcal{O}}$, and

(iii) $\mathcal{O}$ is the power series ring $\bar{\theta}\left[\left[x_{1}, \ldots, x_{s}\right]\right]$.

We note that it follows from (ii) and (iii) that, $\operatorname{dim} \bar{\theta}=\operatorname{dim} \theta-s$. In the special case in which $\theta$ is reduced, $\bar{\theta}$ must also be reduced and hence, if $s=\operatorname{dim} \theta$, then $\bar{\theta}$ must be a product of fields.

THEOREM 12. Suppose $A$ is a reduced analytical $k$-algebra of characteristic zero satisfying Condition II. Let $q$ be a prime ideal in $A$ and let $p=q \cap R$. ( $R$ is the ring discussed in the beginning of this section.) Then the following are equivalent.

(i) $A_{q}$ is integrally closed.

(ii) $A_{q}$ is a regular ring.

(iii) $A_{q}$ is unramified over $R_{p}$.

Proof. We shall first show how (i) implies (ii) and also (iii). Let $d_{1}, \ldots, d_{r}$ be the derivations in $\operatorname{Der}_{k}\left(A, A^{\prime}\right)$ and $x_{1}, \ldots, x_{r} \in A$ the elements arising from Condition II. Let $R=k\left[\left[x_{1}, \ldots, x_{r}\right]\right]$.

Consider the commutative diagram of inclusions.

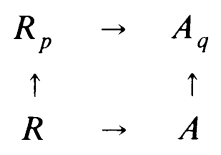

If $A_{q}$ is integrally closed, then $A^{\prime} \otimes_{A} A_{q}=A_{q}$ and it follows that since $A_{q}$ is flat over $A$,

$$
\operatorname{Der}_{k}\left(A, A^{\prime}\right) \otimes_{A} A_{q} \cong \operatorname{Hom}_{A}\left(\Omega_{A / k}, A^{\prime}\right) \otimes_{A} A_{q} \cong \operatorname{Der}_{k}\left(A_{q}, A_{q}\right)
$$

Hence, the derivations $d_{1}, \ldots, d_{r}$ extend to regular derivations on $A_{q}$ (i.e. $d_{i}\left(A_{q}\right) \subset$ $\left.A_{q}\right)$ and generate all the $k$-derivations on $A_{q}$ since the $d_{i}$ 's freely generate $\operatorname{Der}_{k}\left(A, A^{\prime}\right)$.

The flatness of $R_{p}$ over $R$ guarantees that

$$
\operatorname{Der}_{k}(R, R) \otimes_{R} R_{p}=\operatorname{Der}_{k}\left(R_{p}, R_{p}\right) .
$$

Since $d_{i}$ when restricted to $R$ gives the partial derivative $\partial / \partial x_{i}, i=1, \ldots, r$, which together generate $\operatorname{Der}_{k}(R, R)$, we see that every $k$-derivation on $R_{p}$ extends to a $k$-derivation on $A_{q}$. (In fact the extension is unique. If $d \mid R_{p}=0$ then $d \mid R=0$ so, by Lemma $7, d \mid A=\sum d\left(x_{i}\right) d_{i}=0$. Therefore $d$ is zero on $A_{q}$ by the quotient formula.) 
Let $\hat{R}_{p}$ and $\hat{A}_{q}$ denote the completion of $R_{p}$ and $A_{q}$ respectively. These completions are taken in respect to the maximal ideal topology. We then have the commutative diagram

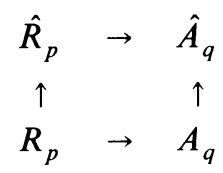

where all arrows are inclusions.

Since taking completions is flat, $\operatorname{Der}_{k}\left(R_{p}, R_{p}\right) \otimes_{R_{p}} \hat{R}_{p} \cong \operatorname{Der}_{k}\left(\hat{R}_{p}, \hat{R}_{p}\right)$ and $\operatorname{Der}_{k}\left(A_{q}, A_{q}\right) \otimes_{A_{q}} \hat{A}_{q} \cong \operatorname{Der}_{k}\left(\hat{A}_{q}, \hat{A}_{q}\right)$.

The same considerations as before allow us to conclude that every $k$-derivation on $\hat{R}_{p}$ extends to a $k$-derivation on $\hat{A}_{q}$.

If $K$ denotes the coefficient field of $\hat{R}_{p}$, then we may assume $k \subset K$ [N, 31.9]. Since $\hat{R}_{p}$ is a regular local ring, $\hat{R}_{p}=K\left[\left[Y_{1}, \ldots, Y_{s}\right]\right]$ where $s=\operatorname{dim} R_{p}$. The partial derivatives $\partial / \partial Y_{i}$ are $K$-derivations on $\hat{R}_{p}$ and, in particular, $k$-derivations which by the above extend to $\hat{A}_{q}$. Denoting these extensions by $D_{i}$, we have exhibited $s$ derivations where $s=\operatorname{dim} \hat{A}_{q}=\operatorname{dim} R_{p}$ and elements $Y_{1}, \ldots, Y_{s}$ in the maximal ideal of $\hat{A}_{q}$ so that $D_{i}\left(Y_{i}\right)=\delta_{i j}$. Theorem 11 now applies and we may conclude that $\hat{A}_{q}=\overline{\mathcal{O}}\left[\left[Y_{1}, \ldots, Y_{s}\right]\right]$ where $\overline{\mathcal{O}}$ is a field since it is local, reduced and of dimension zero. Hence, (i) implies (ii).

The same conclusion shows that $\left\{Y_{1}, \ldots, Y_{s}\right\}$ generate the maximal ideal of $\hat{A}_{q}$ and since these elements already generate the maximal ideal of $\hat{R}_{p}$, it follows that $\hat{p} \hat{A}_{q}=\hat{q} \hat{A}_{q}$. But $p \hat{R}_{p}=\hat{p} \hat{R}_{p}$ and $q \hat{A}_{q}=\hat{q} \hat{A}_{q}$ and so the flatness of $\hat{A}_{q}$ over $A_{q}$ now shows $p A_{q}=q A_{q}$. Since char $k=0$, it follows immediately that $A_{q} / q A_{q}$ is separable algebraic over $R_{p} / p R_{p}$. Hence, (i) also implies (iii).

If (iii), then since $R_{p}$ is regular its maximal ideal is generated by $s=$ $\operatorname{dim} R_{p}=\operatorname{dim} A_{q}$ elements which by assumption generate the maximal ideal of $A_{q}$. It is well known that this implies that $A_{q}$ is a regular local ring [AM, 11.22]. Hence (iii) implies (i). Of course, that (ii) implies (i) is immediate. Q.E.D.

The theorem shows that if $A$ satisfies Condition II, then the singular locus of $A$ and the ramification locus of $A$ over $R$ agree and that this is the same as the primes containing the conductor $C$. In particular, $\sqrt{J}=\sqrt{C}$, and since $C$ contains a regular element so must $J$ under the assumption that $A$ satisfies Condition II. The depth of $C$, i.e. the longest $A$-regular sequence in $C$, is always bounded by 1 [L1]. We now show that if $A$ is also a domain and $A / M=k$ is algebraically closed, then codim $C$ (i.e. the minimal height of any prime containing $C$ ) and hence codim $J$ is always bounded by 1 . We do this by appealing to the ramification locus of $A$ over $R$ and showing that if we assume $A$ is singular, then there must exist primes $q$ in $A$ of height one which are ramified over $R$. This result is known for complex analytic spaces. We prove it by using another theorem by Zariski which we will have occasion to use again later.

If we assume $A$ satisfies Condition II and $R=k\left[\left[x_{1}, \ldots, x_{r}\right]\right]$ as before with quotient field $Q(R)$, then the integral closure $R^{\prime}$ of $R$ in $Q(A)$ is $A^{\prime}$. Referring to the notation before Lemma 3, if we set $R_{i}=e_{i} R$ then $A_{i}^{\prime}$ is the integral closure of $R_{i}$ in 
$F_{i}$ and $A_{i}^{\prime}$ is finitely generated over $R_{i}$. Therefore $F_{i}$ is finite separable (char $k=0$ ) over $Q\left(R_{i}\right)=e_{i} Q(R)$ where $Q\left(R_{i}\right)$ is the quotient field of $R_{i}$.

Any derivation $d$ on $R$ to $R$ may be viewed as a direct sum of derivations $e_{i} d$ from $R_{i}$ to $R_{i}$. The derivation $d$ has an extension $d^{\prime}$ of $R^{\prime}$ to $Q(A)$ which may be viewed as the sum of the extension along the components. Our separable assumption assures this extension $d^{\prime}$ is unique.

THEOREM 13 [Z2, THEOREM 3, P. 525]. Let $R=k\left[\left[x_{1}, \ldots, x_{r}\right]\right]$ be a power series ring over a field $k$, char $k=0$, and let $K$ be the quotient field of $R$. Let $F=F_{1} \oplus \cdots \oplus F_{h}$ $\supset K$ be a direct sum of fields $F_{i}=e_{i} F$ with $F_{i}$ a finite separable algebraic extension of $e_{i} K$. Let $R^{\prime}$ be the integral closure of $R$ in $F$. Let $d$ be a derivation on $R$. Assume that if $p^{\prime}$ is any prime ideal of height one in $R^{\prime}$ which is ramified over $p=p^{\prime} \cap R$, then there exists an element $x \in p$ so that $x \notin p^{(2)}$ and $d x=0$.

Under this assumption, if $d^{\prime}$ is the (necessarily unique) extension of $d$ to $F$, then $d^{\prime}\left(R^{\prime}\right) \subset R^{\prime}$.

THEOREM 14. Suppose $A$ is a complete local domain containing its residue field $k$ which we assume is algebraically closed and of characteristic zero. If A satisfies Condition II and is presumed to be singular, then the codimension of the singular locus is one. (By Theorem 12, this is equivalent to claiming that codim $C=1$.)

Proof. Let $K$ and $F$ be the quotient fields of $R$ and $A$ respectively and let $R^{\prime}$ be the integral closure of $R$ in $F$. By Theorem 12 we need only show that some ht. 1 prime $q$ in $A$ is ramified over $R$. If this is not so, that is if all the ht. 1 primes in $A$ are unramified over $R$, then so are all the ht. 1 primes in $R^{\prime}$. For if $Q$ is a ht. 1 prime in $R^{\prime}$ and if $q=A \cap Q$, then $A_{q}$ is regular, hence integrally closed and therefore $A_{q}=R_{Q}^{\prime}$ since $R^{\prime}$ is also the integral closure of $A$ in $F$. Hence, $Q$ is also unramified over $R$.

By Zariski's result quoted above, we may extend the derivations $d_{1}, \ldots, d_{r}$ from $R$ to $R^{\prime}$. The existence of the elements $x_{1}, \ldots, x_{r}$ so that $d_{i}\left(x_{j}\right)=\delta_{i j}$ assures that $R^{\prime}$ is a regular local domain by Theorem 11. In fact

$$
R^{\prime}=L\left[\left[x_{1}, \ldots, x_{r}\right]\right] \quad \text { where } L \text { is a field. }
$$

But $R=k\left[\left[x_{1}, \ldots, x_{r}\right]\right]$ and therefore $L$ is algebraic over $k$ since the extension is integral. Hence, $R=A=R^{\prime}$ which is contrary to our assumption. Q.E.D.

We remark that if $A$ is described as in the theorem but if we presume $k$ is not algebraically closed, then Condition II does not force codim $J \leqslant 1$.

In fact consider the extensions of rings

$$
Q[[t, x]] \rightarrow Q[[\sqrt{2} t, t, \sqrt{2} x, x]] \rightarrow Q(\sqrt{2})[[t, x]]
$$

where $t$ and $x$ are indeterminates, and $Q$ the rationals. Let $A=Q[[\sqrt{2} t, t, \sqrt{2} x, x]]$, $R=Q[[t, x]]$ and $R^{\prime}=Q(\sqrt{2})[[t, x]]$. Then the quotient ring of $A$ is $Q(\sqrt{2})((t, x))$ and agrees with that of $R^{\prime}$ and $R^{\prime}$ is the integral closure of $R$ in that quotient field. We claim

(i) $A$ satisfies Condition II. This because if we restrict $\partial / \partial t$ and $\partial / \partial x$ from $R^{\prime}$ to $A$, then these are $Q$ derivations on $A$ to $A^{\prime}$ so that

$$
\partial t / \partial t=1, \quad \partial x / \partial x=0, \quad \partial t / \partial x=0, \quad \partial x / \partial x=1 .
$$


If $M$ is the maximal ideal of $A$, then $M^{2} \subset(x, t)$ and it follows that $x$ and $t$ is a system of parameters for $A$. By Lemma 7 , it follows that the derivations generate $\operatorname{Der}_{Q}\left(A, A^{\prime}\right)$.

(ii) The conductor of $A^{\prime}$ to $A$ is $M$ since $M \cdot \sqrt{2} \subset A$. Hence, by Theorem 12, the singular locus is $M$ which is of height 2 .

If we assume $A$ is reduced and $k$ is algebraically closed, then under the assumption that $A$ satisfies Condition II we "might as well" assume $\operatorname{codim} J \leqslant 1$.

COROLlary 15. Suppose $A$ is a reduced analytic $k$-algebra of characteristic zero with $k$ algebraically closed and $A$ satisfies Condition II. Assume that $\operatorname{dim} A>1$ and that $\operatorname{codim} J>1$. Then each of the irreducible components of $A$ are regular local rings. In particular, $A^{\prime}$ is a regular ring.

Proof. Let $p_{1}, \ldots, p_{h}$ be the minimal primes of $A$ and let $A_{i}=A / p_{i}$. We have seen in Lemma 6 that, since $A$ satisfies Condition II, so does the domain $A_{i}$. Our assumption that $k$ is algebraically closed implies by Theorems 14 and 12 that the conductor $C_{i}$ of $A_{i}$ is either of $\operatorname{codim} C_{i}=1$ or that it is all of $A_{i}$ which would mean that $A_{i}$ is integrally closed, hence regular.

If $\bar{C}$ is the image of $C$ in $A / p_{i}$, then $\bar{C} \subset C_{i}$. Let $\bar{p}$ be any minimal prime of $C_{i}$ with preimage $p$. Then $p \supset C+p_{i}$ and it follows that the height of $p>1$ by our assumption. Since $p_{i}$ is an associated prime of $A$, ht. $\bar{p}>1$. Hence, $\operatorname{codim} C_{i}>1$ and the corollary is proved. Q.E.D.

If $A$ is as above, $\operatorname{dim} A>1$, it follows from the corollary that its integral closure is regular (i.e. the integral closure of the components are regular). But if $A$ is reduced and of dimension 1, its integral closure is always regular. Hence, a reduced isolated singularity, defined over an algebraically closed field which satisfies Condition II and is complete must have a regular integral closure.

We now reintroduce the condition that $A / \sqrt{J}$ is a regular ring of dimension $r-1$ where $r=\operatorname{dim} A$. Hence, $J$ is a height one prime ideal.

THEOREM 16. Suppose $A$ is a reduced analytic $k$-algebra where char $k=0$. Suppose $A$ satisfies Condition II and that $A / \sqrt{J}$ is a regular ring of dimension $r-1$ where $r=\operatorname{dim} A$. Then $A^{\prime}$ is a regular ring.

Proof. Theorem 10 assures the existence of a system of parameters $x_{1}, \ldots, x_{r}$ which are part of a system of local coordinates and $k$-derivations $d_{1}, \ldots, d_{r}$ from $A$ to $A^{\prime}$ so that $d_{i}\left(x_{j}\right)=\delta_{i j}$. Furthermore, by Theorem $10 A / \sqrt{J}=k\left[\left[\bar{x}_{1}, \ldots, \bar{x}_{r-1}\right]\right]$ where $\bar{x}_{i}$ is the image of $x_{i}$ in $A / \sqrt{J}$. Let $R=k\left[\left[x_{1}, \ldots, x_{r}\right]\right]$. We have

$$
R \stackrel{i}{\rightarrow} A \rightarrow A / \sqrt{J} \rightarrow 0
$$

where $i$ is the inclusion map. Since $R$ is a unique factorization domain, it follows that the height one prime ideal $R \cap \sqrt{J}$ is generated by the irreducible element $\left(x_{r}-\Phi\right)$ for some $\Phi \in k\left[\left[x_{1}, \ldots, x_{r-1}\right]\right]$. Furthermore, $\left(x_{r}-\Phi\right) \notin\left(x_{r}-\Phi\right)^{(2)}$.

Set $D_{i}=d_{i}+\left(\partial \Phi / \partial x_{i}\right) d_{r}=d_{i}+d_{i}(\Phi) d_{r}$ for $i=1, \ldots, r$. These are $k$-derivations regular on $R$. Since $d_{r}(\Phi)=0, D_{i}\left(x_{r}-\Phi\right)=d_{i}\left(x_{r}\right)-d_{i}(\Phi)+d_{i}(\Phi)=0$ for $i=$ $1, \ldots, r-1$. 
If now $q$ is any height one ramified prime in $A^{\prime}$, then Theorem 12 shows that $A \cap q \supset J$ and since $\sqrt{J}$ is a height one prime, $A \cap q=\sqrt{J}$. It follows that $q \cap R=\left(x_{r}-\Phi\right)$. Theorem 13 now shows that $D_{i}, i=1, \ldots, r-1$, may be extended to regular $k$-derivations on $A^{\prime}$. Since $D_{i}\left(x_{j}\right)=\delta_{i j}$, for $i, j=1, \ldots, r-1$ and char $k=0$, it follows by Theorem 11 that $A^{\prime}=\bar{\theta}\left[\left[x_{1}, \ldots, x_{r-1}\right]\right]$ with $x_{1}, \ldots, x_{r-1}$ analytically independent over $\bar{\theta}$ and where $\bar{\theta}$ may be identified with $A^{\prime} /\left(x_{1}, \ldots, x_{r-1}\right)$. It follows that $\bar{\theta}$ is reduced since it is a subring of $A^{\prime}$ and one dimensional by Theorem 11 . Since $A^{\prime}$ is integrally closed and $x_{1}, \ldots, x_{r-1}$ are analytically independent, $\bar{\theta}$ is also integrally closed. It then follows that $\bar{\theta}$ and, therefore, $A^{\prime}$ is regular. Q.E.D.

We note that under the additional assumption that $k$ is algebraically closed we have

COROLlaRY 17. Suppose $A$ is a reduced analytic $k$-algebra where char $k=0$ and $k$ is algebraically closed. If $A$ satisfies Condition II and if $A / \sqrt{J}$ is a regular ring, then $A^{\prime}$ is a regular ring.

Proof. We may presume that $J$ is a proper nonzero ideal of $A$. Now if $\operatorname{codim} \sqrt{J}>1$, then Corollary 15 gives the result. If $\operatorname{codim} \sqrt{J}=1$, the above theorem gives the result. Q.E.D.

\section{REFERENCES}

[A1] S. S. Abhyankar, Local analytic geometry, Academic Press, New York, 1964.

[A2] _ A remark on the nonnormal locus of an analytic space, Proc. Amer. Math. Soc. 15 (1964), 505-508.

[AM] M. F. Atiyah and I. G. MacDonald, Introduction to commutative algebra, Addison-Wesley, Reading, Mass., 1969.

[B] R. Berger, R. Kiehl, E. Kunz and H.-J. Nastold, Differentialrechnung in der analytischen Geometrie, Lecture Notes in Math., vol. 38, Springer-Verlag, Berlin and New York, 1967.

[DF] R. Draper and K. Fischer, Derivations and small $C_{4}$, Commutative Algebra: Analytic Methods, Series in Pure and Appl. Math., Dekker, New York, 1982.

[L1] J. Lipman, Free derivation modules on algebraic varieties, Amer. J. Math. 87 (1965), 874-898.

[L2] _ On the Jacobian ideal of the module of differentials, Proc. Amer. Math. Soc. 21 (1969), $422-426$.

[N] M. Nagata, Local rings, Interscience, New York, 1962.

[Nc] D. G. Northcott, An introduction to homological algebra, Cambridge Univ. Press, New York, 1962.

[Si] A. Simis, When are projective modules free?, Queen's Paper in Pure and Appl. Math., No. 21, 1969.

[S] J. Stutz, Analytic sets as branched coverings, Trans. Amer. Math. Soc. 166 (1972), 241-259.

[W] H. Whitney, Local properties of analytic varieties, Differential and Combinatorial Topology, S. Cairns (Ed.), Princeton Univ. Press, Princeton, N. J., 1965.

[Z1] O. Zariski, Studies in equisingularity. I. Equivalent singularities of plane algebroid curves, Amer. J. Math. 87 (1965), 507-536.

[Z2] Studies in equisingularity. II. Equisingularity in codimension 1 (and characteristic zero), Amer. J. Math. 87 (1965), 972-1006.

Department of Mathematics, George Mason University, Fairfax, Virginia 22030 